\title{
Multi-parametric Analysis for Atrial Fibrillation Classification in ECG
}

\author{
Ivaylo Christov $^{1}$,Vessela Krasteva ${ }^{1}$, Iana Simova ${ }^{2}$, Tatiana Neycheva ${ }^{1}$, Ramun Schmid ${ }^{3}$ \\ ${ }^{1}$ Institute of Biophysics and Biomedical Engineering, Bulgarian Academy of Sciences, Sofia, Bulgaria \\ 2 Acibadem City Clinic Cardiovascular Center, Sofia, Bulgaria \\ ${ }^{3}$ Signal Processing, Schiller AG, Baar, Switzerland
}

\begin{abstract}
This study participates in the PhysioNet/CinC Challenge 2017 dedicated to the discrimination of atrial fibrillation (AF) from Normal sinus rhythm (Normal), other arrhythmia (Other) and strong noise using single short ECG lead recordings. Our Matlab entry applies multi-parametric AF classification based on: noise detection; heart rate variability analysis (HRV); beat morphology analysis after robust synthesis of an average beat and delineation of $P, Q R S, T$ waves; detection of atrial activity by the presence of a $P$-wave in the average beat and $f$-waves during TQ intervals. A Linear discriminant classifier is optimized by maximization of the Challenge F1 score, adjusting the prior probabilities of 4 classes and stepwise selection of a non-redundant feature set. Top-5 features, which contribute to $>90 \%$ of $F 1$ score are 3 HRV features, $P$-wave presence and mean correlation of all beats against the average beat. On the blinded test set, our entry has F1 score: 0.89 (Normal), 0.85 (AF), 0.67 (Other), 0.80 (Overall).
\end{abstract}

\section{Introduction}

Atrial fibrillation (AF) is the most common cardiac arrhythmia, and is the major risk factor for death, stroke, hospitalization, heart failure and coronary artery disease $[1,2]$. It affects about $2-3 \%$ of the population in Europe [3]. The prevalence of AF increases with age (from about $0.14 \%$ of younger $<49$ years old, to about $14 \%$ of older $>80$ years old) and gender (male to female ratio is 1.2:1).

$\mathrm{AF}$ appears as a result of reentry within multiple circuits in the atria and reflects the electrocardiogram (ECG) with the occurrence of irregular multiform fibrillatory f-waves. Those f-waves are present in overall ECG, but are masked by the high amplitude QRS and T waves, thus could be observed only in TQ intervals, predominantly in $\mathrm{V} 1$, and occasionally in the peripheral leads. The extremely low, reaching to zero amplitudes of f-waves, makes the AF detection very difficult.
The AF detection is based on a single or multiple analyses for the presence of arrhythmia [4,5], rapid heart rate (HR), presence of $\mathrm{f}$-waves in the isoelectric TQ interval $[4,6,7]$ and absence of P-waves [8,9].

Some authors are paying attention to the noise that accompanies the ECG and its impact on the AF detection algorithms. Oster and Clifford [5] are analyzing the performance of the $\mathrm{AF}$ detection algorithms as a function of the QRS detection performance, RR interval irregularity, P-wave absence, f-waves existence, and in presence of noise. They are showing a linear decrease of the AF detection accuracy with reduction of the signal-tonoise ratio. Christov et al. [4] are reporting a false positive detection of their 'wave rectification method' in the presence of electromyographic (EMG) noise, and a false negative detection after EMG filtering.

The 2017 PhysioNet/CinC Challenge [10] provides the ground for competitive improvement of $\mathrm{AF}$ detection algorithms with extensive application: easily accessible single lead ECG; short analysis interval (10-60s); reliable AF discrimination from a broad range of sinus rhythms and non-AF arrhythmias; rejection of potentially unreliable classification in the presence of strong noises. This study participates in the Challenge, aiming to explore the feasibility of multi-parametric AF classification based on: noise detection; heart rate variability (HRV) analysis; beat morphology analysis after robust synthesis of an average beat and delineation of P, QRS, T waves; detection of atrial activity by the presence of a $\mathrm{P}$-wave in the average beat and $\mathrm{f}$-waves during the TQ intervals.

\section{Challenge database}

The Challenge provides a dataset with short single lead ECG recordings [10], including 8528 ECGs (training) and 3658 ECGs (hidden test) for scoring of 4 classes:

1) Normal sinus rhythm (Normal) - 5050 cases $(59 \%)$

2) Atrial fibrillation (AF) - 738 cases $(9 \%)$

3) Other arrhythmia (Other) - 2456 cases (29\%)

4) Too noisy to be classified (Noise) - 284 cases (3\%). 


\section{Method}

The multi-parametric algorithm for analysis of the Challenge data is implemented in Matlab (MathWorks Inc.) as an off-line based processing of the available single channel ECG in its full-length (10-60s). The first analysis step is the QRS detector with a Noise correction feedback, followed by 3 feature extraction blocks: HRV analysis, average beat morphology analysis, analysis of fwaves. The calculated set of $>40$ features is subjected to a Linear discriminant classifier of 4 arrhythmia categories. Details for each analysis block are presented below.

\subsection{QRS detection and Noise correction}

The QRS detector based on Pan\&Tompkins algorithm [11] is relying on the energy threshold from the ECG slope, amplitude and width. Certain ECG characteristics block the correct performance of the QRS detector, therefore a noise correction is activated in cases of:

- Low amplitude ECG: causes QRS detector failure (no QRS is detected). The signal is stepwise amplified until the detector starts to register QRS.

- High-frequency and high amplitude noise: causes a false positive QRS detection during the noise, followed by a temporal $(>2 s)$ block of QRS detector. The noise immunity is improved by zeroing the signal during $\pm 300 \mathrm{~ms}$ around the detected noise and then the QRS detector is restarted. If $\mathrm{RR}$-intervals $>2 \mathrm{~s}$ are still present, they are excluded from the RR-interval series.

\subsection{HRV analysis}

HRV is one of the most robust signs of AF. Therefore, we respected all standard HRV measurements according to the ESC/NASPE Task Force [12]. The frequency domain measurements are, however, not considered due to potentially inaccurate spectrum calculation from the limited RR-interval series available within the short Challenge ECG recordings (10-30s).

RR-Tachogram: 7 features are measured for the series of all RR-intervals as: mean value (RRmean), median value (RRmedian), standard deviation (RRstd), mean deviation (RRmeand), proportions of the standard and mean deviation from the mean value (RRstd\%, RRmeand\%), ratio of mean-to-median value (RRrat).

dRR-Tachogram: 9 features are measured for the series of all RR-interval first differences as: mean value (dRRmean), standard deviation (dRRstd), median deviation (dRRmedian) and their proportions to RRmean (dRRmean\%, dRRmedian\%, dRRstd\%), proportion of $\mathrm{RR}$ intervals differing by $>50 \mathrm{~ms}$ from the preceding $\mathrm{RR}$ interval (PNN50), square root of the mean squared differences of successive RR intervals (RMSSD) and its proportion to meanRR (RMSSD\%).
RR-Histogram: HRV Triangular Index is used to count the total number of RR intervals divided by the number of RR intervals in the modal bin, usually reported after resampling to $128 \mathrm{~Hz}$ (sampling interval $7.8125 \mathrm{~ms}$ ).

Poincaré Plot: 3 features are describing the geometry of the Poincaré plot $\left[\mathrm{RR}_{\mathrm{n}}, \mathrm{RR}_{\mathrm{n}-1}\right]$ :

- Short-to-long term HRV is calculated as the ratio SD1/SD2, where SD1 and SD2 are the minor and the major semi-axes of the fitted ellipse [13].

- Variability in the temporal structure (CCM) quantifies the point-to-point (dynamic) variation of the Poincaré plot [14].

- The correlation coefficient (corRR) represents the linear fitting of all points $\left[\mathrm{RR}_{\mathrm{n}}, \mathrm{RR}_{\mathrm{n}-1}\right]$.

The presented example (Figure 1b) shows obvious HRV plot differences between Normal rhythm and AF.

\subsection{Average beat morphology analysis}

Average beat calculation: The beat morphology is evaluated in a window [-300ms; $0.6 *$ meanRR] around the QRS fiducial point. A robust average beat is calculated by signal-averaging of the most sustained beats with peak-topeak amplitudes in the range mean \pm standard deviation of all beats in the recording. The rejected outliers are suspected as artifacts or abnormal beats with nonsustained amplitudes (Figure 1a).

Cross-correlation analysis: The morphologies of all beats are compared against the average beat by maximal cross-correlation (corBeat). Statistics of corBeat as a mean value, $25 \%, 50 \%$ percentiles of all beats evaluates the influence of atypical morphologies in the recording.

Detection of fiducial points ( R, S, Q, J, T-end, Tpeak, P-peak): The two peaks $(\mathrm{R}, \mathrm{S})$ are distinguished as the most positive and negative extremities in a window of $140 \mathrm{~ms}$ around the QRS fiducial point. The detection of Q, $\mathrm{J}, \mathrm{T}$-end, T-peak is adopted from our previous study [15]. P-peak is searched as the maximal amplitude deflection in the interval (Q-330ms; Q). P-peak is valid if found in a physiologically reasonable interval (Q-280ms; Q-30ms). All detected fiducial points are shown in Figure 1c.

Calculation of intervals (QRS, PQ, QT): $Q R S=J-Q$, $\mathrm{PQ}=\mathrm{Q}-\mathrm{Ppeak}, \mathrm{QT}=$ Tpeak-Q.

Calculation of amplitudes (QRS, J, T, P): QRS peak-to-peak is reported. The amplitudes of $\mathrm{J}, \mathrm{T}, \mathrm{P}$ points are calculated against the offset of the isoelectric $\mathrm{Q}$ point.

Detection of P-wave: $\mathrm{P}$-wave is present if:

- P-peak is found in (Q-280ms to Q-30ms) interval.

- P-peak sign $=$ sign of the R-peak.

- P-peak slope $( \pm 10 \mathrm{~ms})>$ thr $1=0.5 \mu \mathrm{V}$.

- P-peak slope $( \pm 20 \mathrm{~ms})>4 * \operatorname{thr} 1=2 \mu \mathrm{V}$.

- P-peak amplitude $>$ thr $2=16 \%$ of QRSp-p amplitude.

The thresholds are optimized for the training Challenge dataset. Detected and not detected P-waves are shown for the average beat of normal rhythm and AF (Figure 1c). 

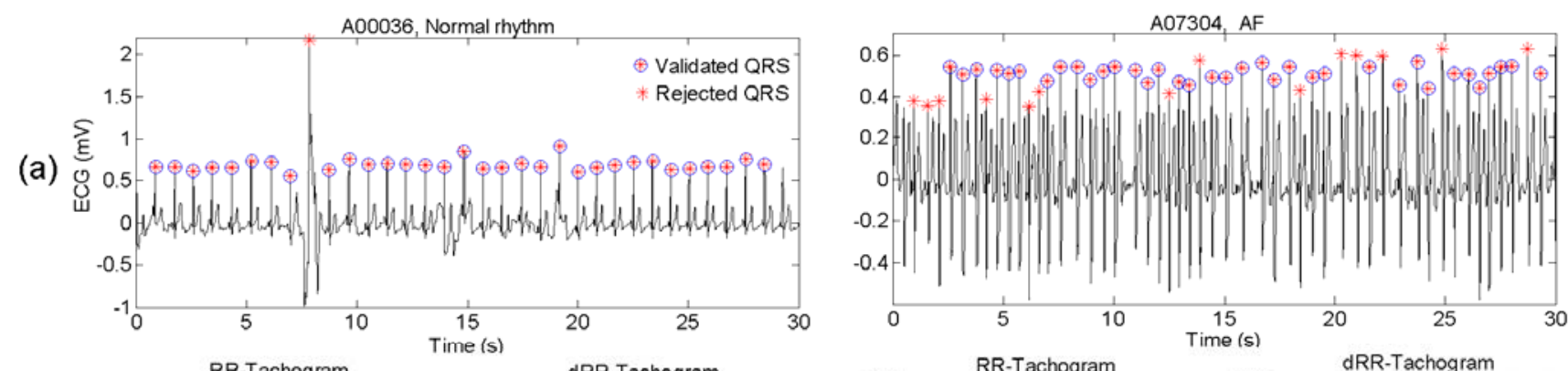

(b)
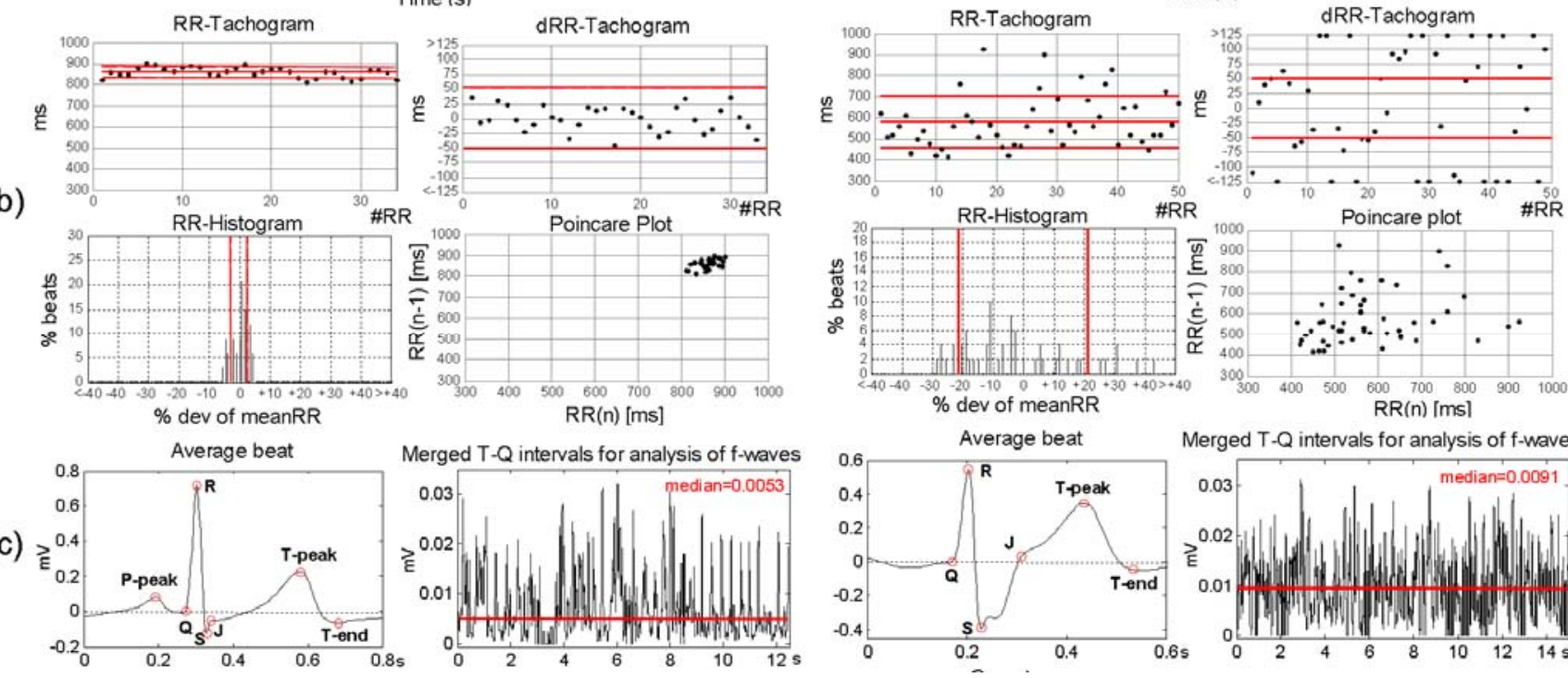

Merged $T-Q$ intervals for analysis of $f$-waves

Figure 1. Two ECG recordings (30s) with annotation 'Normal rhythm' (left) and AF (right) are used to illustrate:

(a) The QRS detection and rejection of non-sustained amplitudes; (b) HRV analysis plots; (c) average beat (left) and the merged T-Q signal for analysis of f-waves (right).
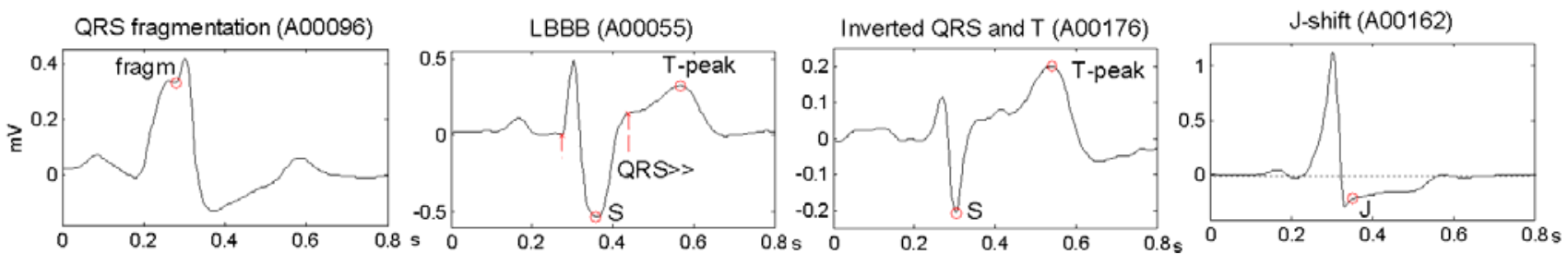

Figure 2. Four examples of average beats with atypical ventricular morphologies.

Detection of atypical ventricular morphologies which are suggested to belong to 'Other' arrhythmia. Figure 2 illustrates four types of detected atypical beats:

- QRS fragmentation: inversion of the slope on the left to the R-peak, which could not be physiologically accepted as Q-point due to the short interval between the slope inversion and the R-peak $(<80 \mathrm{~ms})$ and small amplitude drop (R-fragm $<30 \%$ of QRSp-p).

- Inverted QRS and T-wave: T wave displacement is opposite to the major deflection of the QRS.

- Left bundle branch block (LBBB): a specific case of inverted QRS and T. It is detected by two additional criteria: wide $\mathrm{QRS}>140 \mathrm{~ms}$, high-amplitude $\mathrm{T}>\mathrm{QRS} / 3$.

- J-shift: Offset of J elevation or depression in respect to the $\mathrm{Q}$ point (absolute and normalized values).
Calculation of curvature: The curvature is defined as $\mathrm{c}=1 / \mathrm{r}$, where $\mathrm{r}$ is the radius of the circle, which best fits signal data $x(t)-m e a n(x(t))$ in the least mean square (LMS) sense. The LMS problem is solved by the expression $\mathrm{a}\left(\mathrm{t}^{2}+\mathrm{x}^{2}\right)+\mathrm{bt}+\mathrm{cx}+\mathrm{d}=0$, subject to the constraint $a^{2}+b^{2}+c^{2}+d^{2}=1$, where $[a, b, c, d]$ are found by the singular value decomposition function in Matlab. The center of the circle has coordinates: $x_{c}=-b / 2 a ; y_{c}=-c / 2 a$ and the radius is calculated as: $r=\operatorname{sqrt}\left(x_{c}^{2}+y_{c}^{2}-d / a\right)$.

In our application, we define $\mathrm{t}=20 \mathrm{~ms}$ to calculate the maximal curvature during $\mathrm{P}, \mathrm{QRS}, \mathrm{T}$-wave, and then to report curvature ratios: $\operatorname{maxc}(\mathrm{QRS} / \mathrm{P})$, $\operatorname{maxc}(\mathrm{QRS} / \mathrm{T})$, $\operatorname{maxc}(\mathrm{T} / \mathrm{P})$, which represent the relative activity of different waves, used to distinguish abnormalities, e.g. small/missing P-waves, extremely large or low T-waves. 


\subsection{Analysis of $\mathbf{f}$-waves}

The analysis of f-waves is based on our previous study $[4,7]$, which rejects all [Q; T-end] intervals, where fwaves are masked by QRS, $\mathrm{T}$ high amplitudes. All isoelectric $\left[\mathrm{T}_{\text {end }} ; \mathrm{Q}\right]$ intervals are merged in a continuous signal, which is subjected to DC filtering $\left(1^{\text {st }}\right.$ difference in $20 \mathrm{~ms}$ ), rectification, and EMG filtering (moving average over $30 \mathrm{~ms}$ ). The median value of the merged TQ signal (Figure 1c) is reported as the level of $\mathrm{f}$-waves presence.

\subsection{Linear Discriminant Analysis (LDA)}

A four-class LDA classifier is optimized by adjusting the prior probabilities of different classes, as well as selection of a non-redundant feature set from the total set $>40$ features. The optimization task is performed by forward stepwise feature selection until maximization of the Challenge F1 score [10].

\section{Results and Discussion}

Three LDA models are trained in respect of the mean HR. For each model, the first selected top-5 features, which contribute to $>90 \%$ of F1 score are listed:

- Bradycardia HR<50bpm: 194 cases (7\% Other, 2\% Normal, $1.2 \%$ AF, $0.2 \%$ Normal); Top-5 features $\{$ RRmean, RMSSD \%, PQ, J-amp, $\operatorname{maxc}(\mathrm{QRS})\}$.

- Normal HR=50-100bpm: 7710 cases (99\% Normal, $67 \%$ AF, $80 \%$ Other, $82 \%$ Noise); PNN50, SD1/SD2, $\mathrm{P}$-wave presence, dRRmean $\%$, corBeat (mean)\}.

- Tachycardia HR>100bpm: 624 cases (32\% AF, 16\% Noise, $13 \%$ Other, $0.3 \%$ Normal); \{PNN50, corBeat (25\% percentile), QRS-width, T-amp, QT\}.

Tables 1,2 show the total performance of LDA models. The maximal score is for Normal and AF. A limitation is the false classification of Other as Normal rhythm (23\%).

Table 1. Confusion matrix on the training dataset.

\begin{tabular}{llllll}
\hline & Normal & AF & Other & Noise & Total \\
\hline Normal & 4573 & 26 & 402 & 49 & 5050 \\
\hline AF & 17 & 609 & 105 & 7 & 738 \\
\hline Other & 564 & 158 & 1672 & 62 & 2456 \\
\hline Noise & 37 & 10 & 35 & 202 & 284 \\
\hline Overall & 5191 & 803 & 2214 & 320 & 8528 \\
\hline
\end{tabular}

Table 2. F1 score of our best Challenge entry. The average/max running time is $10 / 11 \%$ of quota.

\begin{tabular}{lll}
\hline & Training set & Blinded Test set \\
\hline Normal & 0.893 & 0.89 \\
\hline AF & 0.789 & 0.85 \\
\hline Other & 0.714 & 0.67 \\
\hline Overall & 0.799 & 0.80 \\
\hline
\end{tabular}

\section{References}

[1] Lip GYH, Fauchier L, Freedman SB, Van Gelder I, Natale A, Gianni $\mathrm{C}$ et al. Atrial fibrillation, Nature Reviews Disease Primers 2 2016;16016.

[2] Camm AJ, Kirchhof P, Lip GY, Schotten U, Savelieva I, Ernst $S$ et al. Guidelines for the management of atrial fibrillation: the Task Force for the Management of Atrial Fibrillation of the European Society of Cardiology (ESC), Eur Heart J 2010;31(19):2369-429.

[3] Zoni-Berisso M, Lercari F, Carazza T, Domenicucci S. Epidemiology of atrial fibrillation: European perspective. Clin Epidem 2014;6:213-20.

[4] Christov I, Bortolan G, Daskalov I. Automatic detection of atrial fibrillation and flutter by wave rectification method. J Med Eng Tech 2001;25(5):217-21.

[5] Oster J, Clifford GD. Impact of the presence of noise on RR interval-based atrial fibrillation detection. J Electrocard 2015;48(6):947-51.

[6] Petrènas A, Marozas V, Sörnmo L, Lukosevicius A. An echo state neural network for QRST cancellation during atrial fibrillation. IEEE Trans Biomed Eng 2012;59(10):2950-7.

[7] Christov I, Bortolan G, Daskalov I. Sequential analysis for automatic detection of atrial fibrillation and flutter. Comp in Cardiol 2001;28:293-6.

[8] Dotsinsky I. Atrial wave detection algorithm for discovery of some rhythm abnormalities, Physiol Meas 2007;28:595-610.

[9] Ladavich S, Ghoraani B. Rate-independent detection of atrial fibrillation by statistical modeling of atrial activity. Biomed Signal Process Control 2015;18(4):274-81.

[10] Clifford GD, Liu CY, Moody B, Lehman L, Silva I, Li Q, Johnson AEW, Mark RG. AF classification from a short single lead ECG recording: The Physionet Computing in Cardiology Challenge 2017. Comp in Cardiol 2017.

[11] Pan J, Tompkins WJ. A real-time QRS detection algorithm. IEEE Trans Biomed Engineer 1985;32(3):230-6.

[12] The Task Force of ESC and NASPE. Heart rate variability. Standards of measurement, physiological interpretation, and clinical use. Eur Heart J 1996;17:354-81.

[13] Golińska A. Poincaré Plots in analysis of selected biomedical signals. Studies in Logic, Grammar and Rhetoric 2013;35(48):117-127.

[14] Karmakar C, Khandoker A, Voss A, Palaniswami M. Sensitivity of temporal heart rate variability in Poincaré plot to changes in parasympathetic nervous system activity. BioMed Eng OnLine 2011;10:17.

[15] Christov I, Simova I. Fully Automated Method for QT Interval Measurement in ECG. Comp in Cardiol 2006;33:321-34.

Address for correspondence.

Ivaylo Christov

Institute of Biophysics and Biomedical Engineering Acad. G. Bonchev str., bl.105, 1113, Sofia, Bulgaria ivohr@biomed.bas.bg 